\title{
Pre-clinical Testing of the Polymer Implants Enriched of Ceramic Materials with aim on Rate of Osteointegration in a Rabbit Model
}

\author{
Lukáš Mitrík ${ }^{1}$, Jana Klímová ${ }^{1}$, Marianna Trebuňová ${ }^{1}$, Marek Schnitzer ${ }^{1}$, Radovan Hudák ${ }^{1}$, Jozef \\ Živčák ${ }^{1}$, Ladislav Molnár ${ }^{2}$, Marcel Modrák ${ }^{1}$ \\ Technical University of Košice, Faculty of Mechanical Engineering, Department of Biomedical Engineering, Automation and Measurement, Letná \\ 9, Košice \\ University of Veterinary Medicine and Pharmacy in Košice, Clinic of Birds, Exotic and Free Living Animals, Komenského 73, Košice
}

\begin{abstract}
The purpose of this paper was writing down the process of preclinical testing which consisted from usage of bioinert polymer material enriched with bioactive ceramic elements. The testing was preceded by a study of the current state and methods of implantation of polymer implants. The created samples were implanted into the animal subject femoral bone with purpose to observe the rate of osteointegration process for twelve weeks. Subsequently were the bones dissected, stripped of soft tissue and observed under stereomicroscope and submitted to histological evaluation. The whole process was detailly recorded and it is prepared for further clinical studies in field of implantation of biocompatible polymer materials.
\end{abstract}

Keywords: animal testing; polymer implants; osteointegration; ceramic elements

\section{Introduction}

Polyether ether ketone (PEEK) belongs to a group of high-performance thermoplastic polymers called PAEK (Polyaryletherketone). Whole group of PAEK polymers is characterized with very satisfying mechanical and chemical properties with wide usage in implantology thanks to their biocompatibility. PEEK in its pure form is highly biologically inert and hydrophobic which lead to zero integration with any biological tissue, its also chemically inert, insoluble in all conventional solvents except $98 \%$ sulfuric acid. The water solubility reaches value $0,5 \%$ of its weight in room temperature. The mechanical value of the modulus of elasticity variates in range of 3-4 GPa where, for example the modulus of elasticity in human cortical bone variates in range of 7-30 GPa. The melting point of pure PEEK material is around $334^{\circ} \mathrm{C}$, for crystallized PEEK material the value changes to $343^{\circ} \mathrm{C}$. All mentioned properties make PEEK material very appropriate option for implants because of its stability in the human body.

Based on clinical application it is possible to classificate PEEK material implants [1, 2, 3, 4]:

1. Bone replacement implants - maxillofacial implants;

2. Implants for spinal surgery - spinal cages;

3. Implants for orthopedic surgery a) lumbar replacements and joint implants, b) fixing devices and screws;

4. Implants for dental surgery - dental prostheses;

5. Implants for cardiac surgery - heart valves, intracardiac pumps.

1.1. Overview of modified PEEK implant structures

Currently there is many scientific papers about different modifications of PEEK

*Corresponding author: Lukáš Mitrík, E-mail address: lukas.mitrik@tuke.sk 
implant structures with aim to improve the individual properties of PEEK material. Below are presented studies from different author's collectives which focused to achieve osteointegration between implants from PEEK material and bone tissue by modifying the implant structures with ceramics materials. $[5,6,7]$

\subsubsection{Utilization of a nano-modified surface on a PEEK implant}

Pär Johansson et al. used in study nanomodified PEEK surface on 48 implants which half of them were coated with nanocrystalline hydroxyapatite $(\mathrm{HA})$ and the other half of implants were left untouched. For the chemical evaluation of the surface was used XPS (PHI 5000C ESCA system, PerkinEImer Inc., Waltham, MA, USA). For the measurement was used micrometer-level surface topography characterized by an interferometer (MicroXAM, ADE Phase Shift, Tucson, AZ, USA). The implants were implanted into 24 testing rabbits manually by performing osteotomy into the rabbit's tibia with aim to reach final diameter of $3,2 \mathrm{~mm}$. Half of the animals were euthanized after 3 weeks of healing and the remaining part after 12 weeks by overdosing with sodium pentobarbital. Subsequently the bones were dissected and removed of soft tissue. During the whole process of healing there were no observations of infection and the implants were successfully removed. The purpose of study was to confirm benefits of usage ceramics material (hydroxyapatite) on polymer implants in process of osteointegration. [8]

\subsubsection{Hydroxyapatite coating on PEEK implants - biomechanical and histological study on rabbit model}

Durham et al. used in study PEEK-PETI-OPTIMA material to create sticks with diameter of $5 \mathrm{~mm}$ and length of $9 \mathrm{~mm}$. The substrates were subsequently abraded with silicon carbide paper and surfaces were rinsed with deionized water to prevent particle contamination. Subsequently were sticks ultrasonically cleaned in acetone, isopropanol and deionized water for 10 minutes, carried out with compressed air and stored in sterile tissue cultures. The sticks were coated with hydroxyapatite (HA)/yttrium-stabilized zirconium (YSZ) by using a rotational base formulation and an ion beam assisted deposition (IBAD) system. For the testing the authors collective used 18 adult male rabbits which were randomly organized into two groups (each contained 9 animals) with different observation time. The first group was observed after 6 weeks and the second one was observed after 18 weeks. In each testing group were implanted 18 coated sticks into the rabbit's femur. The results were obtained by using micro-CT screening device which showed that the biggest bone growth trend was between sixth and eighteenth week after implantation. This information was compared with non-coated PEEK implants and the results were significantly different in field of bone growth and ongoing osteointegration in favour of implants coated with ceramics materials (HA/YSZ). [9]

\section{Experimental Section}

Based on the information gathered from various studies the authors collective from Technical University of Košice carried out the case study with usage of PEEK material enriched with ceramics materials such as hydroxyapatite (HA) and tricalcium phosphate (TCP) in different percentage proportions. The case study was preceded by production of custom filament structures which were subsequently implanted into rabbit femurs with purpose to observe impact of ceramics materials on process of osteointegration.

\subsection{Preparation and production of filaments}

The filaments were produced with cooperation of Slovak Technical University in Bratislava, Faculty of Chemical and Food Technology, Department of Plastics, Rubber and Fibers. The production process consisted from delivery of various polymer pellets from PEEK material enriched with ceramics (Lehvoss Group, Germany) which were used to extrude four different types of composite filaments by TwinScrew extruder (Labtech Engineering, Thailand) with approximated diameter of $1,75 \mathrm{~mm}$.

Type of created filaments:

\section{Sample contained $100 \%$ of PEEK material.}

2. Sample contained $85 \%$ of PEEK material, $15 \%$ of TCP material. 3. Sample contained $85 \%$ of PEEK material, $15 \%$ of HA material. 4. Sample contained $80 \%$ of PEEK material, $15 \%$ of TCP material, $5 \%$ of HA material.

\subsection{Usage of electron microscopy}

All extruded filaments were submitted to electron microscopy for confirmation of the distribution and occurrence of bioactive ceramic elements. For observation was used microscope SECOM SR (Delmic, Netherlands).

\subsection{Methodology and preclinical testing}

The methodology and preclinical testing were prepared and executed with cooperation of 


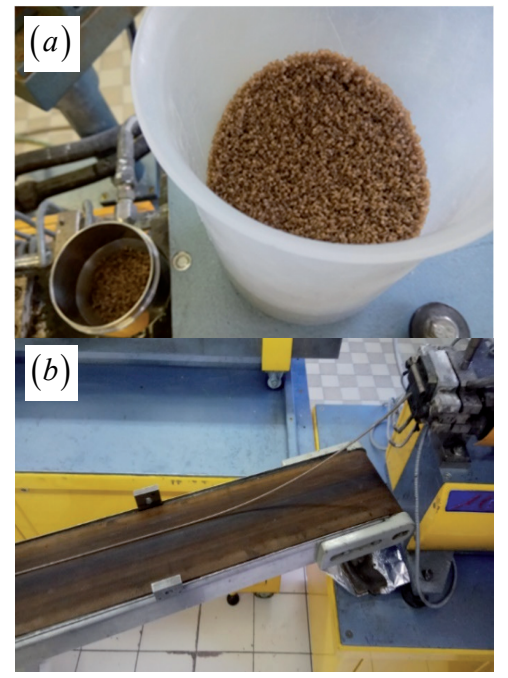

Figure 1: (a) Pellets from PEEK material enriched with ceramics (b) Extrusion of filament.

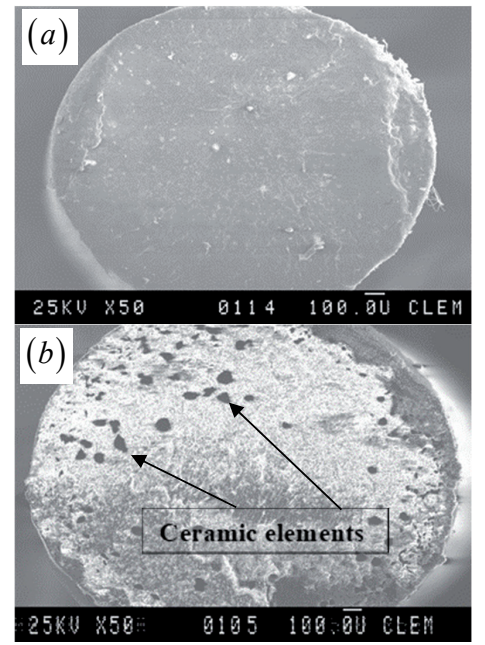

Figure 2: (a) Filament consist of $100 \%$ PEEK material (b) Filament consist of $80 \%$ PEEK material, $15 \%$ of TCP material, $5 \%$ of HA material.

University of Veterinary Medicine and Pharmacy in Košice. As a testing subject was used an adult rabbit with approximate weight of $4,5 \mathrm{~kg}$. The whole surgical procedure consisted of drilling four holes into the rabbit femurs (two holes into each femur) with diameter of $2 \mathrm{~mm}$ which were subsequently implanted with created filaments. The observations were realized after second and tenth week.

\subsubsection{Surgical insertion of the filaments}

The preparation of the rabbit began by using a $1 \mathrm{ml}$ of Cepetor anesthetic $(1 \mathrm{mg} / \mathrm{ml}$, CP-Pharma Handelsgesellschaft mbH, Burgdorf, Germany) representing a half dose due to a surgical surgeon's recommendation. Subsequently it was given $1 \mathrm{mg}$ of Butorfanol (1 mg/ml, Torbugesic ${ }^{\circledR}$, Torbutrol ${ }^{\circledR}$, Dolorex ${ }^{\circledast}$, Laboratories Pharmavet SA, Santa Fe, Argentina), Ketamidor 1,2 mg (100 mg/ml, Richter Pharma Veterinär, Wels). The incision was performed along the proximal tibial epiphysis and marigold cranial was exposed. Gradually, using a drill with a drill bit diameter of $2 \mathrm{~mm}$, two holes were drilled into the both femoral bones. Before the holes was implanted with PEEK filaments with various admixtures were all samples sterilized according to STN EN ISO 11140-1 Sterilization of healthcare products in order to avoid any side effects and infections.

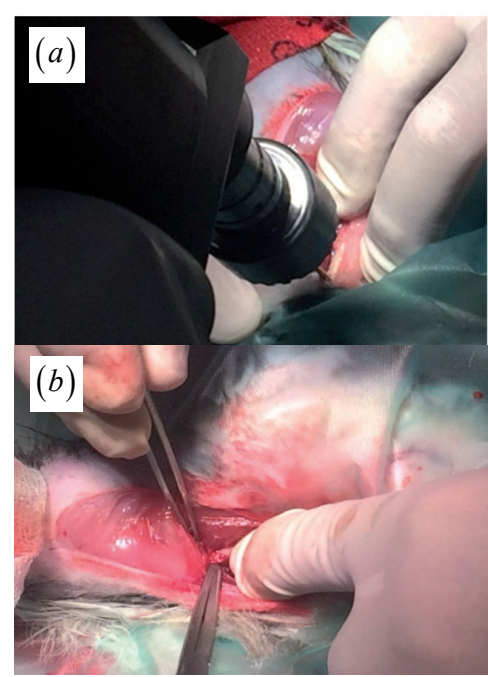

Figure 3: (a) Drilling the holes in rabbit femur (b) Insertion of created filaments.

\section{Results and Discussion}

The whole healing process which lasted for 12 weeks was without any change in health status in testing subject as well as no infection was observed. After twentieth week the rabbit was euthanized and the bones were dissected, removed of soft tissue and prepared for histological testing. Figures presented below shows the position of implanted filaments. In structures which were enriched of ceramic materials occurred successful osteointegration and the implants were bonded to the bone. However, the filament which consisted from pure PEEK material dropped out from the drilled hole due to bioinert properties of PEEK material which lead to non-integration with bone tissue. The removed bones were also observed with 
Stemi DV4 stereomicroscope and video camera AxioCam ERc5s at 20x magnification (Carl - Zeiss, Germany).

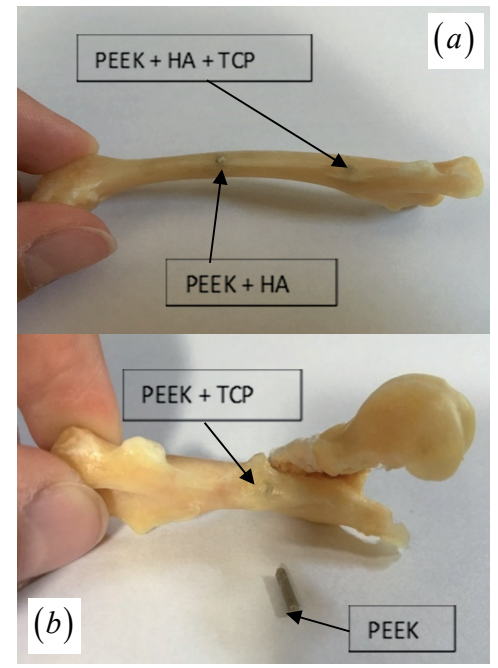

Figure 4: (a) Removed left femoral bone (b) Removed right femoral bone.

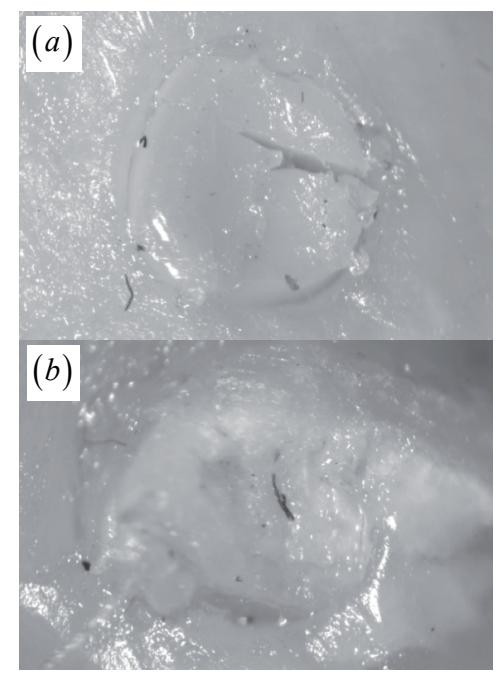

Figure 5: (a) Filament consisting of PEEK and HA material (b) Filament consisting of PEEK + HA + TCP material.

For the full confirmation of the osteointegration process were the samples submitted to the histological analysis which were made with cooperation of Pavol Jozef Šafárik University in Košice, Faculty of Medicine, Department of Forensic Medicine. The bones were decalcified in a decalcification solution for 10 days prior to histological examination, followed by paraffin blocks prepared and truncated on a sledge microtome. The histological sections were stained with Haematoxylin-eosin (HE stains). Evaluated in a Motic BA310 light microscope under magnification of 40x, 100x and 200x.

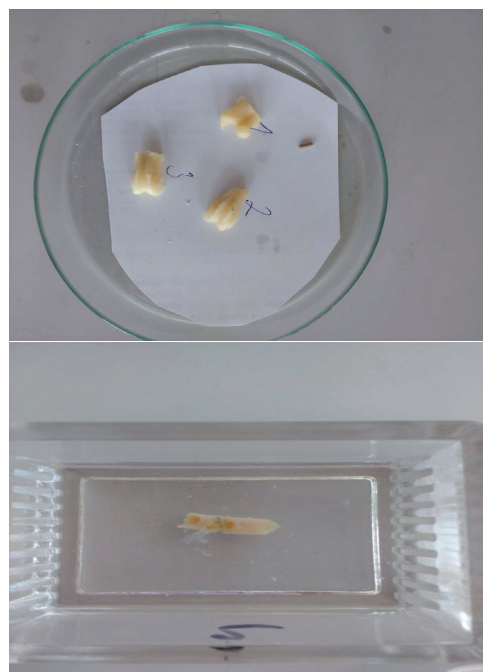

Figure 6: (a) Cut pieces of the bones (b) Bone in decalcification solution.

The histological examination proved that the osteointegration process was successful however, it was not possible to determine if the osteointegration process was increased by bioactive elements in polymer material because the bones were observed after twelve weeks and the highest increase rate of osteointegration is between third and sixth week especially in such small animals like rabbit. Based on acquired knowledge it is necessary to repeat whole case study again with at least two testing subjects which will be divided into two separated groups and the first testing subject will be proceed to histological observation after three weeks and the second one will be observed after six weeks. Such method should provide enough information about the rate of osteointegration process which will be evaluated for further clinical study.

\section{Conclusions}

The paper provides whole process of preclinical examination of custom-made filaments created from polymer material enriched of ceramics elements. The purpose of the presented case study was to determine the rate of improvement of osteointegration process by usage of bioinert 
polymer material combined with the bioactive ceramic materials. The testing was realized on animal subject while the whole methodology, operational and post operational approaches were completely recorded. The study is supplemented of stereomicroscope observations and histological examinations.

\section{Acknowledgments}

This work was supported by the Agency under contract no. APVV15-0356 and no. APVV-17-0278. Project: S-19-103 / 0001-00. Incentives for research and development of the Ministry of Education, Science, Research and Sport of the Slovak Republic: KEGA 023TUKE-4/2020 and KEGA 041TUKE-4/2019.

\section{References and Notes}

1. Panayotov, V I, Orti, V, Cuisinier, F, Yachouh, J, (2016). Polyetheretherketone (PEEK) for medical applications. Journal of Material Science: Materials in Medicine, 27, 118.

2. Shah, A M, Jung, H, Skirboll, S, (2014). Materials used in cranioplasty: a history and analysis. Journal of Neurosurgery, 36, E19.

3. Kurtz, M S, (2012). Peek Biomaterials Handbook (New York: William Andrew).

4. Wang, R, Cheng, K, Advincula, R, Chen, Q, (2019). On the thermal processing and mechanical properties of 3D-printed polyether ether ketone. MRS Communications, 9, 1046-1052.

5. Deng, Q X, Ou, Y S, Zhu, Y, Zhao, Z H, Liu, B, Huang, Q, Du, $X$, Jiang, D M, (2016). Clinical outcomes of two types of cages used in transforaminal lumbar interbody fusion for the treatment of degenerative lumbar diseases: $\mathrm{n}-\mathrm{HA}$ / PA66 cages versus PEEK cages. Journal of Materials Science: Materials in Medicine, 27, 102.

6. Walsh, W R, Pelletier, M H, Bertollo, N, Christou, C, Tan, C, (2016). Does PEEK/HA Enhance Bone Formation Compared with PEEK in a Sheep Cervical Fusion Model? Clinical Orthopaedics and Related Research, 474, 2364-2372.

7. Lee, J H, Jang, H L, Lee, K M, Baek, H R, Jin, K, Hong, K S, Noh, $J$ H, Lee, H K, (2013). In vitro and in vivo evaluation of the bioactivity of hydroxyapatite-coated polyetheretherketone biocomposites created by cold spray technology. Acta Biomaterialia, 9, 6177-6187.

8. Johansson, P, Jimbo, R, Kjellin, P, Currie, F, Chrcanovic, B R, Wennerberg, A, (2014). Biomechanical evaluation and surface characterization of a nano-modified surface on PEEK implants: a study in the rabbit tibia. International Journal of Nanomedicine, 9, 3903-3911.

9. Durham, J W 3rd, Montelongo, S A, Ong, J L, Guda, T, Allen, M J, Rabiei, A, (2016). Hydroxyapatite coating on PEEK implants: Biomechanical and histological study in a rabbit model. Materials Science and Engineering: C; Materials for Biological Applications, 68, 723-731. 